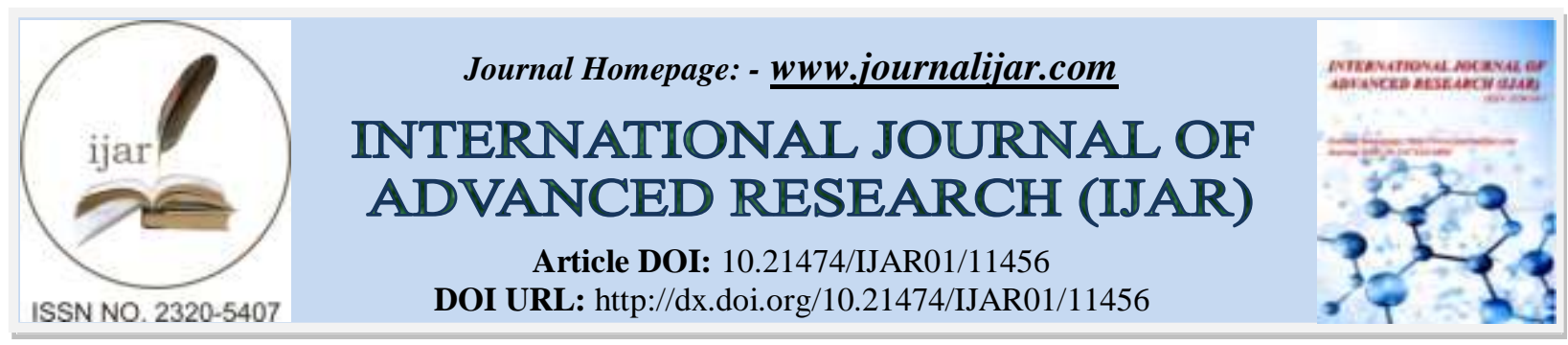

RESEARCH ARTICLE

\title{
QUANTUM DOTS: SYNTHESIS, PROPERTIES AND APPLICATIONS
}

\author{
Dr. Ajita Pathak \\ ECE Department, Amity University.
}

\section{Manuscript Info \\ Manuscript History \\ Received: 31 May 2020 \\ Final Accepted: 30 June 2020 \\ Published: July 2020}

Key words:-

$\mathrm{PbS}$ QDs, QLED, Quantum

Confinement, Wearable Devices,

Flexible Electronics Etc

\begin{abstract}
The size dependent property arising from quantum dot confinement effect leads the way towards many promising applications including flexible electronics, optoelectronics, biomedical, etc. Synthesis of certain $\mathrm{QDs}(\mathrm{PbS})$ remains challenging due to their high reaction rate. Since size and chemical composition are tuneable, thus the properties of these QDs may be controlled and used over wide range of applications in various fields. In this paper the synthesis PbS quantum dot is discussed along with the important characteristics and applications in various fields. Moreover, the advantages of QLEDs in the field of display electronics are discussed which lays foundation for advance wearable devices. The application of QDs which they offer in medical field including detection and treatment of various categories of cancers, with almost negligible side effects is also covered here in this article.
\end{abstract}

Copy Right, IJAR, 2020,. All rights reserved.

\section{Introduction:-}

Quantum dot technology being very useful to various field of science is point of interest of researchers and is being expanded to bring out its applications for mankind. Quantum dots are nanocrystals of semiconducting material with diameter ranging between 2-10 nanometers, which obey quantum mechanical rules of quantum confinement. In other words, emission and absorption spectra corresponding to an energy band of quantum dot is explained by quantum confinement principles. Lower is the size of QDs higher is the band gap of energy band (1,2). Application of QDs increases with display of unique electronic properties lying between bulk semiconductor and discrete molecules. Bigger the size of the crystal, lower is the energy required to excite the dot and less energy is released during return time to the ground state. This results in colour variation from blue to red. Therefore, the variation in size of the QD (3) of same material enables it to emit light of any colour. Figure 1 shows the variation of splitting of energy levels and hence the colour of emitted radiation with variation in size of quantum dots. The fluorescence is the unique feature that makes important use of QDs in biomedical and electronic applications. According to the structure and composition QDs can be classified as: core type quantum dot, core shell quantum dot and alloyed quantum dot. Core type quantum dot possess single component material with uniform internal composition, e.g. $\mathrm{CdTe}, \mathrm{PbS}$ etc. In order to reduce nonradiative recombination and to increase the fluorescence output, one of the methods used is to grow shells of higher band gap semiconductor around lower band gap semiconductor. These quantum dots which possess one lower band gap semiconductor embedded in another with wider band gap are categorised as core-shell quantum dots, e.g. CdSe in core offering band gap of (1.7- 1.76) ev and $\mathrm{ZnS}$ (3.8-4.1) ev, in the shell which are II-VI semiconductors and type I QDs. Shell provides isolation between surrounding and core which give rise to 


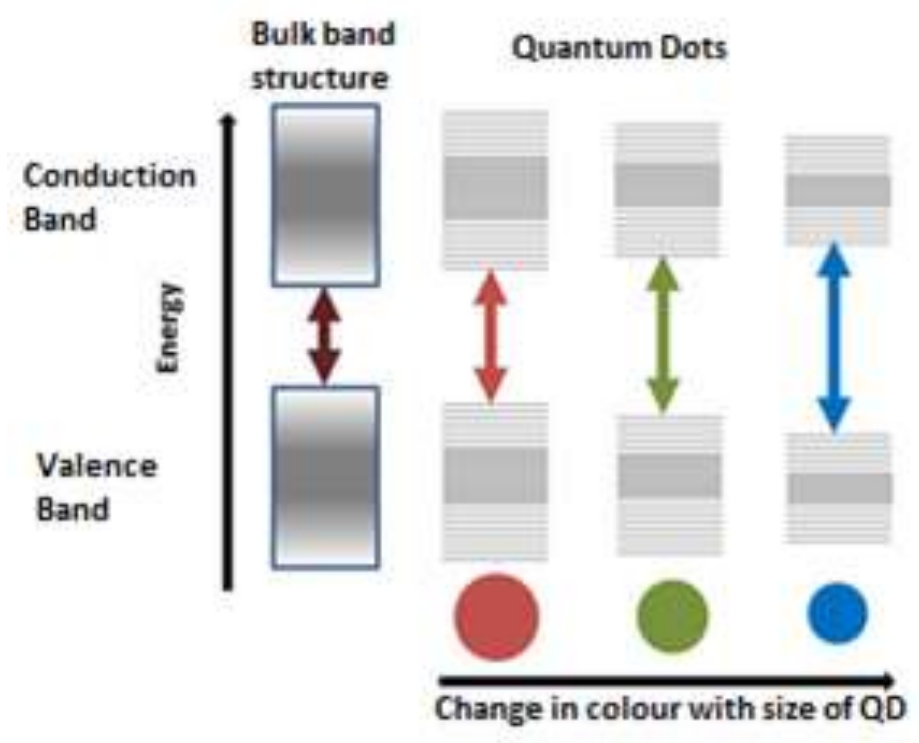

Figure 1. Splitting of Energy Levels

enhanced fluorescence quantum yield. Although the properties of QD can be regulated by their size but crystalline size may cause problem in certain application. The alternate solution to such problem is multicomponent quantum dots i.e alloyed semiconductor QDs. Optical as well as electronic properties may be changed by varying the composition and structure keeping crystalline size same. Such QDs are formed by two different semiconductor materials possessing different band gap energies and thus exhibit distinct characteristics those of their parent semiconductors.

\section{Synthesis of Size Controlled QDs}

There are number of techniques to prepare the QDs as per the requirement $(4,5,6)$. PbS QDs are most suitable for fundamental studies for quantum confined systems. Samples may be used to study strong near infrared photoluminescence. $\mathrm{PbS}$ QDs can be prepared by degassed evacuation for $10-12$ minutes of $\mathrm{PbO}(.45 \mathrm{~g}, 2 \mathrm{mmol})$, oleic acid $(1.3 \mathrm{~mL})$ and octadecene $(49.2 \mathrm{~mL})$ and flushed with nitrogen. This process is repeated for 50 minutes while stirring. Now the mixture is heated under nitrogen to $200^{\circ} \mathrm{C}$ for nearly one hour. TMS $(100 \mu \mathrm{L}$ dissolved in $12 \mathrm{~mL}$ of 1-octadecene) is injected in the hot solution to initiate the nucleation. Mixture is cooled to room temperature and is washed few times by with anhydrous acetone and dispersed in hexane. The absorption peak at $1344 \mathrm{~nm}$ predicts the estimated diameter to be $4.5 \mathrm{~nm}$.

\section{QD Applications}

Composition tuneable electronic properties and nanoscale unique size enables quantum dots to be useful in variety of new technologies $(7,8)$. Fields benefited are: Optoelectronic, Electronics, Biomedical, etc.

\section{Optoelectronic devices \\ Lasers:}

InAs QDs are point of attraction of researchers as they offer number of applications, for example QD ensembles are used in laser active regions. 3-D quantum confinement gives rise to unique properties to quantum dot semiconductor lasers, like: low threshold current density, high temperature characteristics and small line width enhancement factor (9). Second property leads to the increased tolerance towards the optical noise. This enables the use of lasers in metropolitan and LAN. Oleic acid capped colloidal PbS QDs proves to be saturable absorber and may be used to produce ultrashort- pulse generation (10). Nonlinear saturable absorption properties of $\mathrm{PbS}$ QDs are utilised in near-infrared pulsed laser and modulators. GaN QD semiconductor lasers are widely used in high-power and high-temperature optoelectronic devices, for example: sensing and optical coherent tomography, industrial manufacturing, military uses, optical communication, medicine etc. 


\section{Solar cells:}

In recent years the research for the utilization of semiconductor nanostructure for solar energy conversion leads the way towards development of high efficiency solar cells $(11,12)$. Since the diameter of QD is very small, therefore the energy levels of an excited electron within the QD are discrete, opposite to that of bulk semiconductor. Moreover, the band gap varies with the size of QD, the energy absorbed by an electron in the QD from high-energy photons may be transferred out of QD through excited electron and the hole may be filled by low energy electron. PbS QDs possess attracting optoelectronic features for better photovoltaic applications, e.g. large Bhor radius and tuneable band gap $(13,14,15)$. One of the draw backs is the presence of trap states and weak charge transfer between QDs which degrades the photovoltaic performance of solar cell $(16,17)$. To overcome this problem a short ligand (1,2-ethanedithiol) approach is used $(18,19,20,21)$.

\section{Electronic devices QD displays:}

It is well known that human eye possesses only three types of colour sensitive cells, i.e red, blue and green. Screens are made by dividing each pixel into red, blue and green sub-pixels. Mixing of these colours produces various other colours. LCDs use white back light which is layered with three coloured filters. They act as subpixels and are covered by liquid crystal shutter to control the amount of each colour of light. This process requires that the light emerging from each sub-pixel should be as narrow as possible in order to achieve precise colour mixing. This can be done accurately by using QDs (22,23). QDs offer broad absorption and sharp emission characteristics and hence can be used as sub-pixels. After being layered over LED backlight QDs effectively converts unwanted wavelengths into pure red, blue or green instead of filtering them out (24). There are two main advantages of use of quantum dot materials. Firstly, these materials possess property to resist moisture and can be used for longer time span. Second high resistivity to moisture avoid use of expensive vacuum evaporation process. New display techniques using QDs consume low power, perform better with high resolution, and more energy efficient. Moreover, QDs may also be deposited on flexible substrates creating a possibility of light and foldable screens.

\section{Wearable QD displays:}

As discussed above the commercial application lies in the display electronics. Moreover, composition tuneable electronic properties with nanoscale size of QDs paves way to flexible wearable electronics (25). Quantum dot light emitting diode offer purity of colour along with narrow bandwidths, high brightness at low operating voltages (26), high energy efficiency, easy processability, etc. Ultrathin thickness of QLED display enables user to make a comfortable contact with the skin without any mechanical deformation (27). The development of eskin has opened the way to sense pressure, temperature, blood oxygen level, heart rate and other parameters of body. Material used must have properties that of human skin of streachibility, flexibility and no damage with bodily movement QLED displays minimal rise in temperature during the operation, offering the safe use against the skin. Professor Dae-Hyeong Kim with his group at Seoul National University demonstrated a passive matrix quantum dot light-emitting diode (QLED) display fully integrated with flexible electronics. Here ultra-thin red, blue green QLEDs display uses a passive matrix structure to show individual pixels. Brightness and luminescence may be controlled by optimizing the shell thickness of QD. Measured data can be seen by the user on the skin attached display itself.

\section{Biomedical applications}

QD finds several biomedical applications: cell targeting, cancer therapy, diagnosis etc.

1. QDs as tag for drug carriers

2. Anti-cancer applications

3. QDs in neuroscience

4. Biomedical imaging

QDs composed of elements of group III-V are more stable as compared of QDs composed of elements of group II-VI. Reason may be the presence of covalent bond instead of ionic bond. A new class of CdSe QDs of size between 1-2 nm and well-defined structure called magic sized nanocrystals (MSN) are point of attraction of researchers. The properties of MSN are high stability, closed shell structure, strong quantum confinement, high surface to volume ratio and different thermodynamically stable structures. 
Researches shows that quantum dots are generally localised in liver, kidney, spleen and lungs (Karabanova LV, Sukhanova TE et al 2008). However, no conclusions have been made yet about its influencing factors. The absorption, distribution, excretion and toxicity depend on factors ensuing inherent physicochemical properties and environmental conditions. The toxic effects of QDs limit their applications in biology and medicine. The important parameters identified for toxicity are presence of charge, concentration, bioactivity of outer coating, and photolytic property of QD. Besides this they find promising use in drug targeting and vivo biomedical imaging. Advances in the field of drug nanocarriers lay the base of progress of nanomedicine. Mechanism of drug delivery at the target depends on the structural properties of nanomaterial (28). Moreover, tagging a conventional medicine carrier on QD serves as photostable fluorescent and helps to target and cure human diseases. QDs have long lasting photo stability and are brighter than other organic dyes.

\section{Conclusion:-}

As discussed above QDs has contributed in almost every field and especially in the area of wearable devices, opto-electronics, drug delivery, targeting, and imaging. Application in flexible electronics leads the way for better diagnosis and cure for various diseases including cancer. Regarding QD solar cells efficiency and stability a lot of work is required to done in future. Further, integrated sensors and the circuitry used with them may be reduced in size to become more compatible with human skin. Moreover, development of high-resolution ultrathin wearables with better photo-air stability (29), is point of attraction of researchers, using technology based on active matrix operation.

\section{References:-}

1. Reimann, S. M.; Manninen, M. Reviews of Modern Physics, 74(4), 1283, (2002).

2. Bawendi, M. C.; Steigerwald, M. L.; Brus, L. E. Annual Review of Physical Chemistry, (1990), 41, 477.

3. Yoffe, A. D. Advances in Physics, (2001), 50(1), 1

4. Lin, S.C.; Lee, L.; Chang, C.H; Shenand, Y,J; Yang, Y. M.; Applied Physics, Lett., 90, (2007)

5. Sharma S, Datta P, Barua K,Karmakar S, "Synthesis, Characterization and Application of PbS Quantum Dots" AIP Conference Proceedings 1147,436 (2009)

6. Huang Z, Zhai G, Zhang Z, Zhang C, Xia Y, Lian L, Fu X, Zhang D and Zhang J, "Low cost and large scale synthesis of $\mathrm{PbS}$ quantum dots wuth hybrid surface passivation" CrystEngComm, Issue 6 (2017).

7. Vahala, K. J. Nature, 424(6950), 839, (2003).

8. Ameenah Al-Ahmadi: Janeza Trdine 9, 51000 Rijeka, Croatia,(2012)

9. H. Al-Husseini, Amin H. Al-Khursan, S. Y. Al-Dabagh, "III-Nitride QD lasers", Open Nanosci J. 3, 1-11, (2009).

10. Ling Yun, Yang Qiu, Conghao Yang, Jie Xing, Kehan $\mathrm{Yu}$, Xiangxing $\mathrm{Xu}$, and Wei Wei, "PbS quantum dots as a saturable absorber for ultrafast laser", Photonic Research,vol. 6 (11), 1028-1032,(2018)

11. S. Rühle, Tabulated values of the Shockley-Queisser limit for single junction solar cells, Solar Energy. 130: 139-147, (2016)

12. Vokhmnintcev K.V., Samokhvalov P.S., Nabiev I.R., "Charge transfer and separation in photoexcited quantum dot-based systems", NanoToday, 11 (2), 189-211, (2016).

13. O. Voznyy, D. Zhitomirsky, P. Stadler, Z. Ning, S. Hoogland, E.H. Sargent, A charge-orbital balance picture of doping in colloidal quantum dot solids, ACS Nano 6, 8448-8455, (2012)

14. M. Graetzel, R.A.J. Janssen, D.B. Mitzi, E.H. Sargent, Materials interface engineering for solutionprocessed photovoltaics, Nat. 488 (2012) 304-312

15. M.A. Hines, G.D. Scholes, Colloidal PbS nanocrystals with size-tunable near-infrared emission: observation of post-synthesis self-narrowing of the particle size distribution, Adv. Mater. 15 (2003) 18441849.

16. M.M. Tavakoli, A. Tayyebi, A. Simchi, H. Aashuri, M. Outokesh, Z. Fan, Physicochemical properties of hybrid graphene-lead sulfide quantum dots prepared by supercritical ethanol, J. Nanoparticle Research 17 (2015) $1-13$.

17. M.M. Tavakoli, R. Tavakoli, P. Davami, H. Aashuri, A quantitative approach to study solid state phase coarsening in solder alloys using combined phase-field modeling and experimental observation, J. Comput. Electron. 13 (2014) 425-431.

18. M.M. Tavakoli, K. H. Tsui, S. F. Leung, Q. Zhang, J. He, Y. Yao, D. Li, Z. Fan, Highly Efficient Flexible Perovskite Solar Cell with AntiReflection and Self-Cleaning Nanostructures, ACS Nano, (2015) DOI: 10.1021/acsnano.5b04284. 
19. Y. Gao, H. Jin, Q. Lin, X. Li, M.M. Tavakoli, S. Leung, W.M. Tang, L. Zhou, H.L. Wa Chan, Z. Fan, Highly flexible and transferable supercapacitors with ordered three-dimensional $\mathrm{MnO} 2 / \mathrm{Au} / \mathrm{MnO} 2$ nanospike arrays, Journal of Materials Chemistry A 3, (2015) 10199-10204.

20. S.G. Shabestari, M.M. Tavakoli, M. Ghanbari, Effect of Semi-Solid Processing on Iron-Bearing Intermetallic Compounds in A380 Aluminum Alloy, Solid State Phenomena, 217 (2015) 397-404.

21. M.M. Tavakoli, S.G. Shabestari, M. Ghanbari, Effect of thixoforming on morphological changes in ironbearing intermetallics and mechanical properties of $\mathrm{Al}-\mathrm{Si}-\mathrm{Cu}$ alloys, International Journal of Materials Research 105 (2014) 1210-1217

22. Dai, Xingliang, et al. "Solution-processed, high-performance light-emitting diodes based on quantum dots." Nature 515.7525 (2014) 96-99.

23. Coe-Sullivan, Seth, et al. "Quantum dots for LED down conversion in display applications." ECS Journal of Solid State Science and Technology 2.2 (2013) R3026-R3030.

24. Jang, Eunjoo, et al. "White-Light-Emitting Diodes with Quantum Dot Color Converters for Display Backlights." Advanced materials 22.28 (2010) 3076-3080.

25. H. Lee, T. K. Choi, Y. B. Lee, H. R. Cho, R. Ghaffari, L. Wang, H. J. Choi, T. D. Chung, N. S. Lu, T. Hyeon, S. H. Choi, D. H. Kim, "A graphene-based electrochemical device with thermoresponsive microneedles for diabetes monitoring and therapy", Nat. Nanotechnol. (2016), 11, 566.

26. B. S. Mashford, M. Stevenson, Z. Popovic, C. Hamilton, Z. Q. Zhou, C. Breen, J. Steckel, V. Bulovic, M. Bawendi, S. Coe-Sullivan, P. T. Kazlas, "A graphene-based electrochemical device with thermoresponsive microneedles for diabetes monitoring and therapy" Nat. Photonics, 7, (2013), 407-412.

27. Jaemin Kim, Hyung Joon Shim, Jiwoong Yang, Moon Kee Choi, Jiwoong Yang, Moon Kee Choi, Dong Chan Kim, Taeghwan Hyeon, Dae-Hyeong Kim, "Ultrathin Quantum Dot Display Integrated with Wearable Electronics", Advanced Materials vol.29(38), (2017).

28. Zhang W, Yao Y, Chen YS. 'Imaging and quantifying the morphology and nanoelectrical properties of quantum dot nanoparticles interacting with DNA'. J Phys Chem C. 115:599-606, (2011).

29. J. M. Caruge, J. E. Halpert, V. Wood, V. Bulovic, M. G. Bawendi, "Colloidal quantum-dot light-emitting diodes with metal-oxide charge transport layers", Nat. Photonics (2008), 2, 247. 